\title{
Smart Lock Systems: An Overview
}

\author{
Diamond Celestine Aluri \\ Dept. of Computer Science and Systems Engineering, \\ Andhra University College of Engineering for Women, \\ Visakhapatnam, Andhra Pradesh, India
}

\begin{abstract}
Smart lock can be regarded as the modern successor of the traditional lock. This paper provides an overview of different smart lock technologies. The smart lock system being adopted in many homes and other commercial buildings today is an innovative approach that provides many user-friendly benefits with its tolerable downsides.
\end{abstract}

\section{Keywords}

Smart lock, mobile apps, sensors, Bluetooth, Home security, Home automation

\section{INTRODUCTION}

Technology today has made a greater influence on many aspects of our life. The Home Automation System is a computerized and automated network of electronic devices fashioned to exhibit control over home appliances and to efficiently monitor them [1]. One of the emerging real-time application that has gained much light is the Home Security System. The smart lock system is an emerging framework that is gradually replacing the traditional locks due to its convenience and affordable prices.

A smart lock is an electromechanical device that performs locking/unlocking operations using wireless protocols. Wired networks were previously replaced by wireless communications as they tend to provide more flexibility and extensibility with an easy installation [1]. The smart lock is operated wirelessly by a cryptographic key on authentication providing access only to the authorized personnel. With the advancement of technology and research, more robust security mechanisms are developed. Accessing the smart lock system using a fingerprint biometric system provides for increased levels of security. Some smart locks have added camera functionality to enhance their security. The smart lock is plausibly the most salient component of a smart connected home.

A smart lock can be used for either commercial (offices, hotels, malls, etc.) or residential (i.e., Home security) security purposes. It allows third-party entities to access the lock utilizing a virtual key that is sent to the recipient smartphone over $\mathrm{Wi}-\mathrm{Fi}$, mobile app, proximity sensors, BLE enabled devices, etc. Integrating Wi-Fi into the smart lock can have several benefits among which is all-time status OTA updates.

The number of smartphone users is rapidly increasing, and its uses are tremendous. Usage of a smartphone or other gadgets to monitor and control the security of domestic or commercial buildings makes the task much easier. It is, however, possible to easily misplace keys than to lose a phone hence, the smart lock system uses this fact to its advantage. Moreover, to ensure high-end security while using smartphones, the standard AES encryption can be used which is hard to penetrate [3].

Various versions of smart locks have been developed and the need to improve security offers a wide area of research. In this paper, few of the versions of smart locks are elaborated with their salient features.

\section{BASIC COMPONENTS}

\subsection{Electromechanical lock}

For the functioning of a smart lock, like the traditional locks, it requires a lock and a key. Here, the electromechanical lock accomplishes locking and unlocking operations employing a cryptographic key that receives instructions using a wireless protocol. It is an electronically augmented deadbolt installed onto any desired location (usually an exterior door) [2].

\subsection{Cryptographic key}

A cryptographic key is either a smartphone or a key fob which authenticates to automatically open the door and is generated by executing an encryption algorithm. The key required to unlock is not necessarily a smartphone now. The technologies have evolved to a larger extent which now implements the locking and unlocking operations via BLE enabled devices, sensors, and various other protocols such as ZigBee, and the like, which are discussed in this paper.

\section{MODES OF WORKING}

\subsection{Mobile/Web apps}

The smart locks affixed provide access to its authorized users through digital keys. The digital keys issued are highly encrypted and can provide access through different levels namely, owner, resident, recurring guest, and temporary guest [2]. These smart locks once installed require the user to login to the associated mobile/web application in order to be authenticated. Security and privacy are regarded as a major concern. The authenticated users can access the smart lock through the application from anywhere with a strong internet connection. The authorization process executes using a wireless protocol and a cryptographic key. These applications are designed in such a way that it provides for alerts to the user's mobile device when any intruder tries to unlock or gains unauthorized access. The owner can grant or revoke access any time to trusted ones via sharing the virtual keys through standard messaging protocols (e-mail, SMS). The owner is provided with access to view all the lock access logs and monitor its entire functioning. The resident keys have alltime access to the home/building whereas the recurring user has scheduled access as set by the owner. The temporary guest also has similar access in a short-term access window [2].

Many locks support pairing up with devices through Bluetooth connectivity (Ultraloq UL3, August 3rd Gen) but, now to have a strong pairing and the longevity of the connection throughout the building many favor robust Wi-Fi connection as well (August Pro 3rd Gen with Connect Wi-Fi Bridge). 


\subsection{Sensors}

There are several home automation devices either in existence or are currently being developed that allow for automated mechanisms to monitor and control devices that are a part of the home security system. In this paper, the smart lock is the major concern that plays a key role in ensuring high security to the users from theft or other criminal activities that might hamper their security or privacy. The idea of embedding sensors into the smart lock ensures an effortless way to lock or unlock your door. Sensors like PIR, optical sensor and such are used to read and detect objects.

The most common motion sensor used in smart locks is the PIR (Passive Infrared) sensor. The PIR sensor measures the infrared light radiating from the objects with the help of electronic semiconductors [1]. All warm-blooded animals induce IR radiation. These sensors comprise a pyroelectric film material that responds to the infrared radiation by generating electricity which triggers the set burglar alarm. They are an effective means for a home security system as they also consume less energy and are almost long-lasting.

A PIR sensor can be combined with a microwave sensor to operate in different areas of the spectrum. The microwave sensor emits microwave pulses to detect the reflection off the mobile objects. Thus, such dual technology motion sensors are very less likely to trigger false alarms. Many recent developments recommend combining video cameras with advanced signal processing that are controlled by motion sensors.

Several optical sensors that are most commonly used for contactless detection are incorporated in smart locks. An optical sensor evaluates the physical quantity of light and transforms it into a readable form for the integrated measuring device based on the associated sensor. Biometric authentication refers to the identification of humans by their characteristics or traits [4]. While implementing biometric authentication for the smart locks, it requires the user to enter the finger through the optical sensor that generates a template of finger images which are compared to match templates from the library. Fingerprint verification involves generating fingerprint templates that are stored as images or as biometric algorithms. This makes it easier to match and verify fingerprints against the pre-specified and stored fingerprints in the libraries. This system offers increased levels of user authentication to provide access only to authorized users.

\subsection{Bluetooth}

Bluetooth is considered as a wireless network solution in the realm of home networking. Bluetooth provides for an elementary, economical, and secure solution which has made its use quite widespread in mobile devices. Bluetooth is a short-range wireless technology standard that aims at the simplified exchange of data among devices through shortwavelength UHF radio waves in the network [5]. With the help of the Bluetooth system, the user can transmit signals to the door to lock or unlock with any BLE device that is available (tablet, smartphone, etc.). This technology facilitates the administrator or owner to send digital signals to other trusted ones to provide access which requires NFC equipped devices.

In general, the users are required to install the lock's mobile app on their smartphones/tablets to have an authenticated user account to prevent unauthorized access and to monitor the functioning of the security system. Once installed, the mobile device is paired with the lock via a local wireless channel
(Bluetooth Low Energy). These locks depend on the user's mobile device which acts as an Internet Gateway which transmits information between the server and the mobile device in the BLE range of the lock. Once the devices are paired, whenever in the BLE range the devices are connected, and the user can utilize all the available functions to control the lock. The BLE enabled devices can operate within a range of $10 \mathrm{~m}$ to $100 \mathrm{~m}$ of distance at the speed of up to $3 \mathrm{Mbps}$ depending on the class of the Bluetooth devices. They operate over a frequency band of $2.4 \mathrm{GHz}$ [6]. It also facilitates sending e-keys over the mobile app and its ability to detect the user's presence is another feature that has increased its usage.

\subsection{Wi-Fi}

Wi-Fi is said to be the family of wireless networking technologies that provide high-speed internet and network connection. It uses radio waves to ensure an easy, secure and quick data exchange between devices over a network. The usage of smartphones has undergone a rapid increase and various applications require a strong internet connection which can be provided by having a robust $\mathrm{Wi}-\mathrm{Fi}$ connection. Smartphones are now also used as a tool to control smart home technology that is growing at a very fast rate.

The smart lock system requires to be connected to a home LAN connection to which the user is connected. The Local Area Connection (LAN) which can be formed by a simple router is said to be the most essential and key component of the smart lock system that is built based on Wi-Fi security. The smart lock system is designed such that it consists of a central control system that performs functions such as reading the commands, storing data, locking/unlocking operations, and other functions that crucial to the system. A mobile application provides the user with all controls to monitor the locking system as he/she desires. It stores all the digital keys and the user can also create and send temporary keys to guests or any trusted ones [3]. This system has an easy installation process and with its operation, by minimum complexity and strong feasibility, it provides for better locking system prospects [3].

Table 1. Summary of the properties of few smart locks (source: "Smart Locks: Lessons for Securing Commodity Internet of Things Devices", by Grant Ho, et al.)

\begin{tabular}{|c|c|c|c|}
\hline Locks & $\begin{array}{c}\text { Interaction } \\
\text { Model }\end{array}$ & Devices & Interface \\
\hline Kevo & $\begin{array}{c}\text { Touch-to- } \\
\text { unlock }\end{array}$ & $\begin{array}{c}\text { smartphone, } \\
\text { key fob }\end{array}$ & $\begin{array}{c}\text { Mobile } \\
\text { app, } \\
\text { website }\end{array}$ \\
\hline August & $\begin{array}{c}\text { button in } \\
\text { mobile app; } \\
\text { automatic } \\
\text { unlocking }\end{array}$ & smartphone & Mobile app \\
\hline Lockitron & $\begin{array}{c}\text { button in } \\
\text { mobile app } \\
\text { or web } \\
\text { interface }\end{array}$ & $\begin{array}{c}\text { Smartphone, } \\
\text { website }\end{array}$ & $\begin{array}{c}\text { Mobile } \\
\text { app, } \\
\text { website }\end{array}$ \\
\hline Dana & $\begin{array}{c}\text { button in } \\
\text { mobile app; } \\
\text { automatic } \\
\text { unlocking }\end{array}$ & Smartphone & $\begin{array}{c}\text { Mobile } \\
\text { app, } \\
\text { website }\end{array}$ \\
\hline
\end{tabular}




\section{FEW EXAMPLES OF SMART LOCK}

\subsection{Teodoor}

The Teodoor lock is a reliable Bluetooth technology lock that uses the latest military-grade encryption technologies. With its cryptographic co-processor that enables the usage of safer encryption algorithms that reduce the processing time, it also lowers the power consumption. It is powered by replaceable lithium batteries. It also works on a hands-free mode where it locks/unlocks the door on detecting its owner. The Teodoor lock facilitates detailed scheduled access functions and also provides an easy and quick way to provide access rights to the trusted ones by the owner. [7]

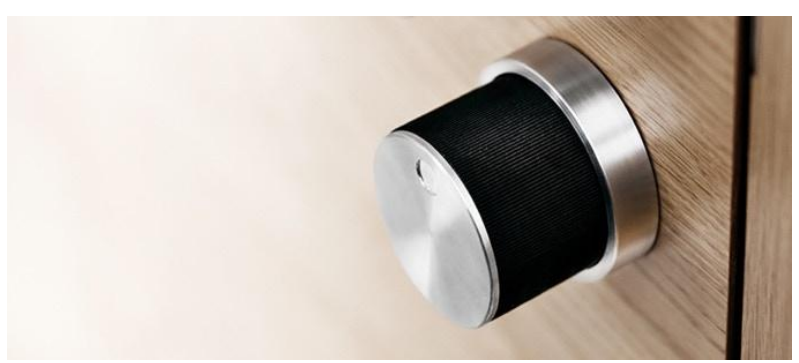

Figure 1: Teodoor Smart Lock (source: https://www.kickstarter.com/projects/1947538842/teodoorsmart-lock)

\subsection{Kevo}

The Kevo smart locks are manufactured by Kwikset which is an American lock and lockset manufacturer owned by Spectrum Brands Hardware and Home Improvement Group, a Division of Spectrum Brands. The Kevo smart lock uses multiple levels of military-grade encryption to provide increased smart lock security. The dual-factor authentication adds extra security to the smart lock system. In case of a lost phone, one can long into their kevo account online to suspend e-keys and disable their phones. Most Kevo locks link the smartphone and the lock using Bluetooth. It allows creating electronic keys that can be shared with the trusted ones. Another important feature is the Kevo fob that can be used in the absence of a smartphone. The Kevo family includes the Kevo Contemporary lock that provides a touch-to-open convenience, the Kevo traditional lock, the Kevo Convert lock that has a smart lock conversion kit, Kevo plus that provides for remote access, and the Kevo fob. [8]

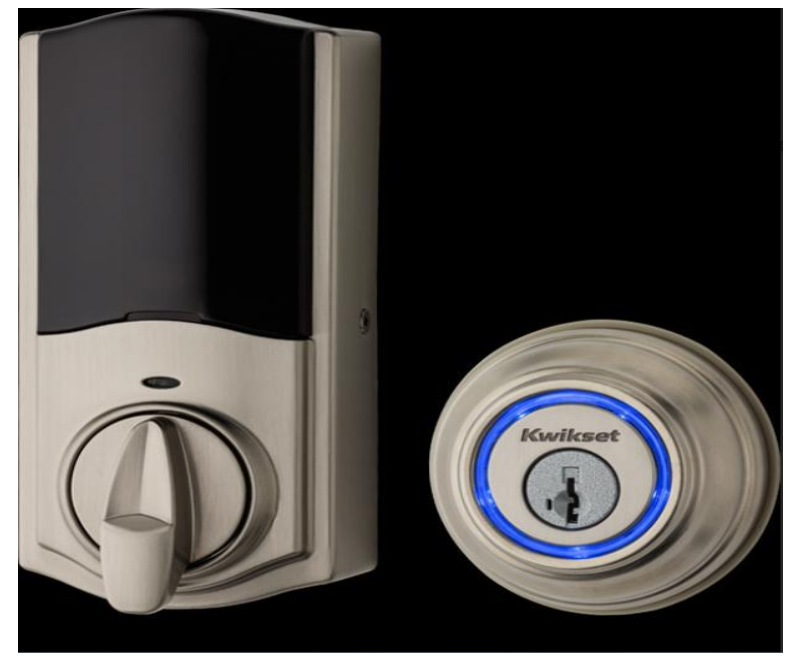

Figure 2: Kevo smart locks from Kwikset (source: https://www.kwikset.com/kevo/smart-lock/security)

\subsection{August}

August has launched its Wi-Fi smart lock which provides remote access to lock/unlock doors, grant virtual keys to trusted ones or use a voice assistant to control it. With its built-in Wi-Fi, it requires no bridge to connect. The smart lock system allows pairing with smartphones or other potential devices to enable keyless access. It requires the owner to be an authorized user on the August app which provides an efficient interface to monitor and control the lock and to check for the status and any updates.

The guest book function to keep track of all the recent access data is an important feature embedded into it as it keeps the owner informed about all the activities to provide an even secure home in a smarter way. The smart lock system by August $\left(1^{\text {st }}\right.$ gen or $2^{\text {nd }}$ gen, etc.) uses strong encryption standards that include BLE encryption technologies and TLS in mobile applications [9].

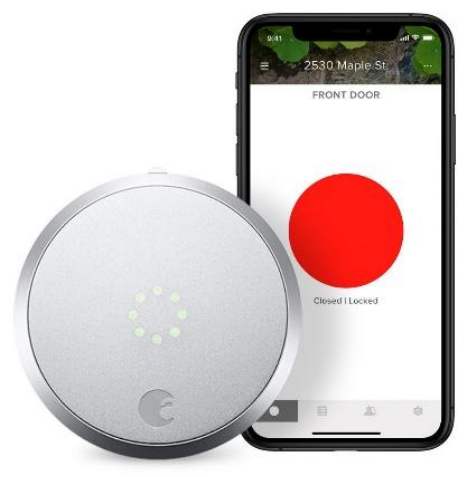

Figure 3: August Smart Lock (source: https://august.com/products/august-wifi-smart-lock)

\section{ADVANTAGES}

The installation of smart locks to enhance the security of homes or any commercial building is made very convenient and easy. Also, the security standards make them a much reliable substitute for conventional locks. This technology implements an automatic locking/unlocking system that can be activated by smartphones or biometric readers which eliminates the dilemma of being locked out or misplaced leys. The key feature of providing exclusive access to authorized users enhances the security and these locks cannot be easily tampered. The functionality to detect intruders or any person and to record their activities in the set geographical range present in many smart lock systems ensures a much reliable and secure smart home. It serves as an ideal tool to ensure security at commercial buildings and work environments as this system allows only authorized personnel to have access control to a specific area. Besides having an easy installation process, it makes it easier to activate and access the lock as it does not restrict it to smartphones; the advanced versions consist of key fobs and other BLE devices that can access the locks simultaneously. Despite having a biometric identification or smartphone access to lock/unlock, many locks now provide a keypad to create a pin or password that identifies the authorized user.

\section{DISADVANTAGES}

The major drawback of smart locks is when the owner tries to access the lock with a smartphone with a low battery. During a low battery condition, the smartphone has many of its features disabled and it may not be efficient enough to 
perform its locking/unlocking function as it might further drain the battery. Also, during a blackout of the control unit or any faulty condition the user is stranded as he/she cannot gain access to the system. Like any software it is possible for intruders to exploit its vulnerabilities and to tamper the device; the manufacturers thus make constant efforts to release regular updates that improve their encryption standards in order to make it harder for unauthorized users to penetrate and to increase the security of the system.

\section{CONCLUSION}

The smart lock system has gained great significance in the global market today. Constant efforts are being made to improve the standards of security and to ease the operation and control over the smart home security system. This paper outlines various features that are currently available in smart locks. Considering the various versions discussed, it gives us an ample understanding of how the smart lock system enhances the security of home or workplace by providing hassle-free access from anywhere.

\section{REFERENCES}

[1] Jayant Dabhade, Amirush Javare, Tushar Gayal, Ankur Shelar, Ankita Gupta 2017. "Smart Door Lock System: Improving Home Security using Bluetooth Technology", International Journal of Computer Applications (0975 8887) Vol. 160 (8), pp.19-22.

[2] Grant Ho, Derek Leung, Pratyush Mishra, Ashkan Hosseini, Dawn Song, David Wagner 2016. "Smart Locks: Lessons for Securing Commodity Internet of Things Devices", in ASIA CCS'16: Proceedings of the 11th ACM on Asia Conference on Computer and Communications Security, pp. 461-472.

[3] Abdallah Kassem and Sami El Murr, Georges Jamous, Elie Saad and Marybelle Geagea 2016. "A Smart Lock System using Wi-Fi Security", in 2016 3rd International Conference on Advances in Computational Tools for Engineering Applications (ACTEA), pp.222-225.

[4] Anu and Bhatia, D. 2014. "A smart door access system using finger print biometric system", Int. J. Medical Engineering and Informatics, Vol. 6, No. 3, pp.274-280.

[5] Stogu Pavel 2015. "Smart Lock System", https://www.theseus.fi/handle/10024/98023

[6] Lubhansh Kumar Bhute, Gagandeep Singh, Avinash Singh, Vikram Kansary, Preetam Rao Kale, Shailendra Singh 2017. "Automatic Door Locking System Using Bluetooth Module", International Journal for Research in Applied Science \& Engineering Technology (IJRASET), Vol. 5 (V), pp.1128-1131.

[7] Martin K, Teodoor, your personal doorman - Kickstarter, $\begin{array}{llll}\text { accessed } & 26 & \text { June } & 2019 \text {, }\end{array}$ <https://www.kickstarter.com/projects/1947538842/teod oor-smart-lock>

[8] Spectrum Brands, Inc. n.d., Kwikset - Kevo, 2019 <https://www.kwikset.com/kevo/smart-lock/security>

[9] August Home, designed in San Francisco, California, 2019, August Wi-Fi Smart Lock <https://august.com/products/august-wifi-smart-lock> 